Recepción: 18 / 05 / 2018

Aceptación: 20 / 07 / 2018

Publicación: 05 / 10 / 2018

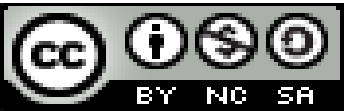

Ciencias de la educación

Artículo de investigación

\title{
Análisis de la problemática de la EIB en la comunidad Cañari de Quilloac, 2014
}

Analysis of the problems of the EIB in the Cañari community of Quilloac, 2014

Análise dos problemas do BEI na comunidade Cañari de Quilloac, 2014

Carmen L. Moreno-Vintimilla ${ }^{\text {I }}$

\section{Correspondencia: carmen.morenov@ucuenca.edu.ec}

I Licenciada en Ciencias de la Educación en la Especialización de Ingles, Docente de la Universidad de Cuenca, Cuenca, Ecuador. 


\section{Resumen}

La presente investigación aborda la problemática de la Educación Intercultural Bilingüe en la comunidad de Quilloac, de la provincia de Cañar, en Ecuador. Desde una perspectiva histórica se abordan hechos que contribuyeron a la creación de nuevos espacios identitarios para las comunidades indígenas de Cañar a través de reformas sociales y del sistema de Educación Intercultural Bilingüe. Se empleó la intervención dialógica para indagar sobre el problema que el Instituto Superior Pedagógico Intercultural Bilingüe-Quilloac (ISPEDIB-Q) afrontaba en el año 2014 frente a su inminente desaparición y la incertidumbre que para la comunidad implicaba este hecho dentro del contexto de las reformas educativas formuladas por el Gobierno Nacional del Ecuador. Se realizó un acercamiento a la realidad de la comunidad mediante un enfoque etnográfico en el que se empleó una metodología cualitativa-descriptiva. El estudio da a conocer cómo se llevaba a cabo la formación de educadores bilingües (kichwa- español) en el Instituto Pedagógico en Quilloac y los factores inherentes a la realidad social del contexto educativo. Este trabajo dio lugar a una serie de cuestionamientos acerca de la pertinencia de las políticas gubernamentales en torno a la EIB del Ecuador y la necesidad de respetar la autodeterminación del pueblo Cañari en sus procesos de formación y en la apropiación de su lengua y su cosmovisión como factores clave para la cohesión de su vida comunitaria y de su identidad cultural.

Palabras clave: educación; interculturalidad; formación docente; Quilloac.

\section{Abstract}

This article analyzes the problematic situation that the community of Quilloac, in Cañar-Ecuador faced during the social reforms that took place within the Intercultural Bilingual Education System. From a historical point of view, there were analyzed some facts that contributed to create new identity spaces for these indigenous communities in Cañar, because of the social reforms that occurred within the Intercultural Bilingual Education. A dialogical intervention was used in order to search the problem that the Pedagogical Bilingual Intercultural Institute - Quilloac (ISPEDIBQ) faced in 2014 because of an imminent disappearance of the Pedagogical Institutes in the country and the uncertainty that this fact implied in the community, within the context of educational reforms formulated by the national government of Ecuador. An Ethnographic 
approach was used in order to get in contact with the community, through a qualitative and descriptive methodology, in order to show how the career of bilingual teachers (kichwa-Spanish) took place in the Pedagogical Institute in Quilloac and its inherent factors of its educational context. This work leads us to a series of questions about the relevance of government policies regarding the EIB of Ecuador and the need to respect the self-determination of the Cañari people in their teaching training process. The respect to the appropriation of their language and culture were also considered as key factors for the cohesion of their community life and their cultural identity.

Key words: education; interculturality; teachers training; Quilloac.

\section{Resumo}

Esta pesquisa trata do problema da Educação Bilíngue Intercultural na comunidade de Quilloac, na província de Cañar, no Equador. De uma perspectiva histórica, os fatos que contribuíram para a criação de novos espaços de identidade para as comunidades indígenas de Cañar através de reformas sociais e do sistema de Educação Intercultural Bilingue são abordados. Dialógica intervenção para investigar o problema do Superior Pedagógico Intercultural Bilíngüe-Quilloac (ISPEDIB-Q) Instituto enfrentou em 2014 contra a sua morte iminente e incerteza para a comunidade envolvida este fato no contexto das reformas foi utilizada atividades educativas formuladas pelo Governo Nacional do Equador. Uma abordagem da realidade da comunidade foi feita através de uma abordagem etnográfica em que foi utilizada uma metodologia qualitativadescritiva. O estudo revela como ele realizou a formação de professores bilingues (kichwaespanhol) do Instituto Pedagógico em Quilloac e fatores inerentes à realidade social do contexto educacional. Este trabalho levou a uma série de perguntas sobre a relevância das políticas governamentais ao redor do BEI do Equador e a necessidade de respeitar a autodeterminação do povo Cañari em seus processos de formação e a apropriação de sua linguagem e visão de mundo como fatores-chave para a coesão de sua vida comunitária e sua identidade cultural.

Palavras chave: educação; intercultural idade; formação de professores; Quilloac. 


\section{Introducción}

El presente estudio analiza el caso de la comuna de Quilloac, localizada en la provincia de Cañar, en Ecuador, y su proceso de creación de un sistema educativo adaptado a las especificidades culturales de una comunidad Kichwa-hablante, a partir de la Reforma Agraria y las consiguientes reivindicaciones políticas y sociales de los pueblos indígenas que tuvieron lugar en Ecuador durante un proceso de cerca de 30 años, entre 1966 y finales del siglo XX. Para este fin se tomará como dato referencial la transición del sistema de hacienda hacia la tenencia de la tierra por parte de los miembros de la comuna, y la formación de la comunidad educativa. Estos hechos históricos crearon nuevas configuraciones culturales en comunidades indígenas como la de Quilloac, en la que, a través de la apropiación de su lengua y de su cultura, se propició el fortalecimiento de su identidad en el contexto de la Educación Intercultural Bilingüe.

En Ecuador, como en otros países de la región, el proceso de Interculturalidad fue un camino para que las comunidades Kichwa-hablantes pudiesen alcanzar, a través de la educación, su reivindicación social y política como una manifestación cultural y linguiística de las minorías indígenas (López, 2002). Se pasó así del sistema homogeneizador mono lingüista del idioma castellano, a un sistema en el que la recuperación de la lengua autóctona cobró importancia a través de los actores sociales de la comunidad. Quilloac optó por ese camino de lucha.

Es importante recuperar las evidencias de los procesos que marcaron esa transformación política y cultural a través de las distintas luchas sociales que se dieron en el transcurso de recuperación de su territorio, al igual que por el derecho vital del agua y por una mejor educación. En todas estas historias se reconoce el espíritu independiente de la gente de Quilloac.

Fue así como la apropiación cultural jugó un papel importante en su lucha por la educación, y a través de ella alcanzaron reivindicaciones culturales y lingüísticas, además de una independencia en cuanto a la organización de su propio sistema educativo, enfocado de manera tal que reflejara su filosofía de vida. A partir de la Reforma Agraria iniciada en 1964, esta comunidad pudo recuperar parte de su territorio y mantuvo su identidad cultural como pilar de la educación; luchó constantemente para tener una educación basada en su propia lengua y cultura, lo que ha constituido una de las reivindicaciones socio-políticas y culturales del pueblo Cañari. "Este hecho ha caracterizado a esta comunidad por su bravura y coraje ayer, hoy y siempre" (Verdugo, 2010). 
La formación docente constituyó para Quilloac una de sus fortalezas en EIB. A través ella, se formó a más de 1000 maestros en las aulas del Instituto Pedagógico desde 1984 hasta 2014, año en que el gobierno, a través de la Secretaría Nacional de Educación Superior, Ciencia, Tecnología e Innovación (SENESCYT), cerró los institutos pedagógicos de formación bilingüe y la Universidad Intercultural de las Nacionalidad y Pueblos Indígenas o Amawta Wasi; entre otras instituciones educativas en donde se impartía una real educación intercultural basada en una filosofía que respondía a la cosmovisión de los pueblos andinos. La formación de maestros en EIB pasó entonces a ser competencia de la Universidad Nacional de Educadores (UNAE), creada exclusivamente para la formación docente, dentro de la denominación Educación Intercultural, que incluye en la actualidad la educación mestiza y bilingüe.

Con estos antecedentes se formularon las siguientes preguntas de investigación:

¿Qué pasaría con los alumnos docentes al cerrarse el Instituto Pedagógico Quilloac y cuál sería el futuro de estos estudiantes?

¿Cómo se desarrollaban sus prácticas docentes y cuál era el manejo de la lengua kichwa y de la segunda lengua, (español) en sus clases?

¿Cómo se concibe a la interculturalidad desde las actuales leyes educativas en el sistema de educación bilingüe, al ser manejadas desde el currículo oficial?

Para este fin se utilizaron varias técnicas de investigación como las entrevistas abiertas a educadores y maestros, la aplicación de cuestionarios, y el registro de historias de vida de los actores involucrados en el proceso de la EIB; todo esto, dentro de un enfoque etnográfico (Guber, 2011). En una segunda fase de investigación realizada en el año 2015 se buscó analizar el proceso de la enseñanza del español como lengua segunda en relación a la lengua kichwa.

La reflexión a partir de estas preguntas trajo a colación cuestionamientos acerca de la factibilidad real de que el nuevo sistema educativo impuesto por el gobierno pudiera mantener una coherencia filosófica con la formación que hasta ese entonces habían recibido los docentes dentro de su comunidad. La creación de una carrera de EIB dentro de los paradigmas linguiísticos de una formación totalmente adscrita al currículo de la lengua española, podría errar en su enfoque al omitir el valor de los rasgos semánticos de la lengua materna, en este caso el kichwa, lengua 
basada en una cosmovisión y filosofía de vida contrapuesta, en muchos aspectos, a la cosmovisión occidental oficializada a través del español y que contradice de raíz lo que Walsh define dentro de la dimensión intercultural como "una realidad transformadora intrínseca, que no responda únicamente al sistema político sino a un sistema de pensamiento existencial, en donde el actor este inmerso en la experiencia cotidiana" (2012: 38).

\section{Historia de la Unidad Educativa Bilingüe Quilloac}
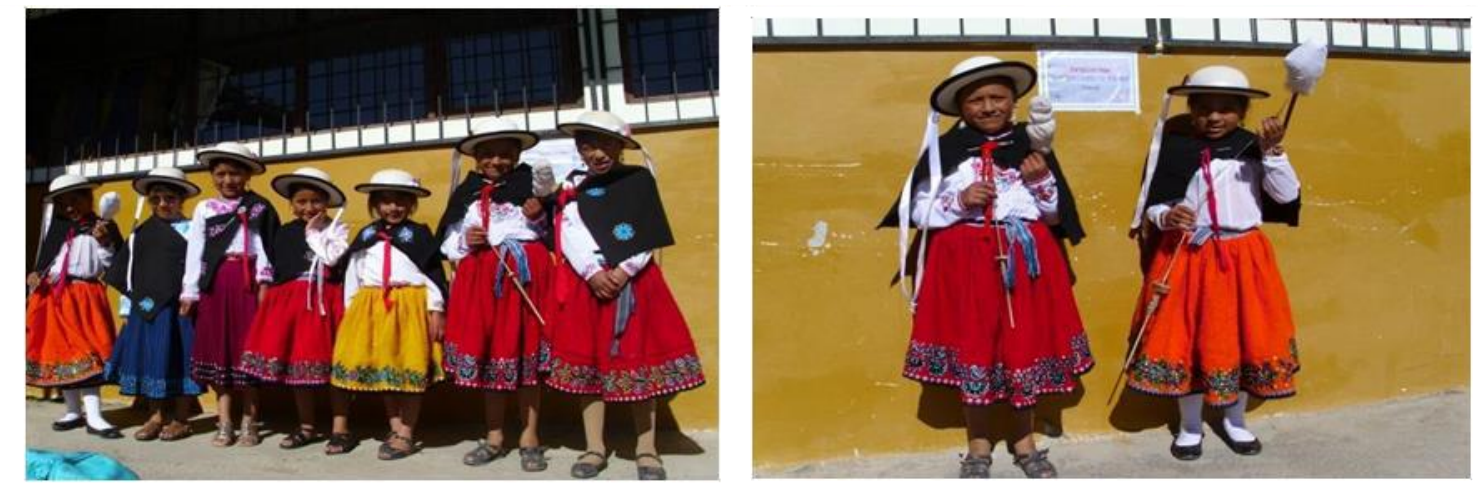

Fuente: fotografía de mi autoría, 2015

La Comunidad de Quilloac está situada en la Provincia de Cañar, en el sur de Ecuador. Caracterizada por su rica historia y cultura, ha sido un lugar de interés y de estudio de varias disciplinas. Arqueólogos, investigadores sociales y educadores han puesto siempre su mirada en este lugar, cuna de la cultura Cañari. Quilloac se asienta en las inmediaciones del Cerro Narrío, región donde habitaron las culturas aborígenes de Narrío, Sizho y Cashaloma, y sitio ancestral donde, hasta hoy en día, se realizan ceremonias y rituales que representan la mitología del lugar.

Luego de la reforma agraria de 1963-64 en Ecuador, se realizó la adjudicación de tierras a los campesinos y, en gran parte, ellos la adquirieron comprándolas. Por la característica de escaso riego disponible en la zona, la comunidad se planteó como una meta mejorar este aspecto mediante los trabajos que fueron realizados por sus propios miembros. Al mismo tiempo surgió como otro aspecto postergado la necesidad de acceder a un sistema de educación formal, que junto con la urgencia por acceder al agua hizo que el objetivo de la comuna se orientara en esas dos direcciones (Guamán, 2011). 
Durante la presidencia del Dr. Jaime Roldós Aguilera (1979-1981) en el país, se dio la creación de la Unidad Educativa Quilloac, conjuntamente con el centro comunal que luego pasó a ser parte de la institución; se conformó así la unidad educativa con el pre-escolar, escuela y colegio. Se creó además el Instituto Educativo de Formación Docente con el fin de preparar al alumnado como futuros maestros en EIB.

Con la Constitución de 2008 surgió la Ley Orgánica de Educación Intercultural Bilingüe (LOEIB), la cual desestructuró la organización de la Unidad Educativa de formación inicial, escuela, y formación docente y pasó a convertirla en Instituto Superior Pedagógico, para que funcionase bajo las regulaciones de la Secretaría de Educación Superior, Ciencia, Tecnología e Innovación (SENESCYT). En la última evaluación realizada entre 2010 y 2011 el Instituto Superior Quilloac ocupó el segundo lugar a nivel nacional con un puntaje de $81.60 \%$, después del Instituto Pedagógico Superior Jaime Roldós, de la provincia de Riobamba. Desde 2010 a 2013 el Instituto Superior Quilloac había sido constantemente evaluado y sus logros fueron exitosos (Quinde, 2014)

Con el apoyo del Consejo de Evaluación, Acreditación y Aseguramiento de la Calidad de la Educación Superior (CEAACES) estos dos institutos han continuado una trayectoria de formación docente en las Provincias de Cañar, Imbabura, Pichincha, Cotopaxi y Santo Domingo de los Tsáchilas, mediante una modalidad semi-presencial para profesores en servicio activo. El Instituto Pedagógico Quilloac ha formado hasta la fecha más de mil docentes, según los datos registrados en 2010 .

\section{Problemática del Instituto Superior Pedagógico Intercultural Bilingüe Quilloac (2014)}

En Latinoamérica la EIB está regida por circunstancias disímiles, dadas en gran parte por las políticas educativas de los diferentes gobiernos, que muchas veces van en detrimento de los logros alcanzados en años de lucha de reivindicaciones por parte de pueblos y comunidades. Es lamentable decir que en Ecuador aún se vive en la práctica un sistema neoliberal que rige nuestro sistema nacional educativo. Se puede criticar abiertamente la forma como es concebida en la actualidad la EIB, hecho que se refleja en el truncamiento de la labor docente del Instituto Superior Pedagógico Intercultural Bilingüe Quilloac (ISPEDIB-Q), en el año 2014. 
En ese tiempo, el ISPEDIB-Q luchaba por mantenerse como tal, pues la formación docente era una necesidad permanente en la provincia de Cañar, con el fin de mantener la enseñanza intercultural bilingüe a través de la sistematización de la lengua kichwa y la valoración de los referentes culturales propios de sus comunidades. En Quilloac, la EIB había sido diseñada sistemáticamente, y organizada desde la atención al proceso de gestación de las madres a través del cuidado de los centros comunitarios de salud. Se programaba la educación bilingüe desde el nivel inicial, en Centros donde se atendía a niños menores de 5 años y a madres y familias con el objeto de prepararlas para que el niño/a venga ya predispuesto al conocimiento de la lengua materna (Guamán, 2011).

En Quilloac la educación formaba parte integral de un contexto social comunitario. Los estudiantes que optaban por seguir la carrera de Educación Superior en el ISPEDIB-Q, se formaban como docentes en EIB. El ISPEIB-Q se constituyó de esta manera como una unidad educativa integrada a la vida cotidiana de la comunidad. El aprendizaje bilingüe empezaba desde el nivel inicial, pasaba por la educación Básica, y posteriormente al Bachillerato, donde los estudiantes que optaban por seguir la carrera de Educación Superior se graduaban como maestros en enseñanza de educación bilingüe, kichwa-español.

La desarticulación de este sistema provocó un retroceso hacia aquello que para Tedesco (2003) se encuentra relacionado con un orden patriarcal, de características totalitarias, que había perdido funcionalidad en las sociedades contemporáneas, dando lugar a que fuera la familia quien asumiera una función de autoridad y de socialización primaria, donde "las responsabilidades individuales están regidas y asociadas a otros flujos y redes que rigen la vida cotidiana” (Ibíd.). Es más, al verse obligado a retornar a un sistema impuesto desde una lógica homogeneizadora de la educación, se retrocede también al tiempo de la escuela como formadora de un sujeto universal, destinado a satisfacer las necesidades de fuerza laboral de la sociedad industrial (Dubet y Martucceli, 1998; cit. en Tenti Fanfani, 2002; Tiramonti, 2008).

La problemática descrita soslaya los derechos de las comunidades a su autodeterminación, partiendo del hecho de la irrupción en su concepto de formación integral como forma de reproducción de su cosmovisión y tradiciones culturales, además de la incertidumbre causada en los casos individuales de los jóvenes que venían formándose con la perspectiva de convertirse en 
maestros, con los riesgos implícitos de la pérdida de un sistema que de manera funcional fomentaba la cohesión social y el orgullo identitario de un pueblo ancestral.

\section{Metodología}

Para el desarrollo de este trabajo fue necesario realizar un estudio etnográfico del contexto en el que se desarrollaba esta investigación, por lo que no se puede obviar el involucramiento del investigador en su interacción social con la comunidad, dentro de lo que se puede definir el ser un observador participante como lo refiere Guber (2011). Se empleó un método cualitativodescriptivo, con la aplicación de herramientas como la entrevista a los directores docentes del ISPEDIB-Q, y el registro de datos sobre escolaridad. De forma adicional, se incursionó en el quehacer de los alumnos-docentes, mediante entrevistas semi- estructuradas con el fin de poder sistematizar el número de aspirantes que desearían continuar su carrera en la universidad; para esto se realizaron preguntas concernientes a la movilidad y a sus lugares de trabajo, como también otras sobre aspectos metodológicos en el aprendizaje de la segunda lengua, lo que serviría de base de análisis para continuar la investigación. Se tomó también como referencia el diario de vida de una alumna docente con el fin de ahondar en la realidad de su trabajo y tomar la información que fuera pertinente para corroborar los datos obtenidos. Todo esto para conocer en una primera fase de la investigación cuál era la realidad de los alumnos durante la práctica docente.

A partir de este primer trabajo, se tuvo la oportunidad de asistir a una conferencia en la que la Directora Nacional de EIB expuso las nuevas políticas de Educación Intercultural Bilingüe que regían a los centros educativos bilingües en el país; por lo que fue factible tomar en cuenta las diferentes versiones de directores, líderes, maestros, docentes en EIB, además de directivos de otros centros educativos de otras comunidades y de estudiantes-docentes del ISPEDIB-Q. Adicionalmente se entrevistó al líder de educación en la Comuna de Quilloac, quien supo informar sobre las ideologías y creencias de la filosofía Cañari, como aporte para conocer mejor su cultura y el contexto de investigación. En la Unidad Educativa se aplicaron también entrevistas a tres maestras, a quienes se abordó con preguntas abiertas sobre su experiencia docente. El corpus fue recopilado a través de conversaciones y toma de notas en turnos. Las entrevistas y transcripción de las notas y el proceso de escritura de la información se realizaron en español. 
Como se puede ver el procedimiento de este análisis exploratorio es en su mayor parte de orden cualitativo y tiene como propósito dar a conocer la realidad del medio comunitario escolar donde estas alumnas se desenvuelven. El método comparativo constante enfocado en la búsqueda de semejanzas y diferencias presentes en los datos sirvió de ayuda en este trabajo.

\section{Resultados}

En el aspecto de formación docente, los datos obtenidos del informe del centro educativo fueron los siguientes: desde 2008 a 2014 funcionó como Instituto Tecnológico y la formación docente se realizaba en ciclos de 3 años. Se incluyó en el cuadro a los alumnos que se graduaron en el mes de junio, 2014. Como se puede notar en el transcurso de 24 años, el Instituto tecnológico había formado a 1082 docentes, lo cual es digno de reconocimiento a nivel del país.

\section{Gráfico 1. Docentes que estudiaron en el ISPEBIQ hasta 2014}

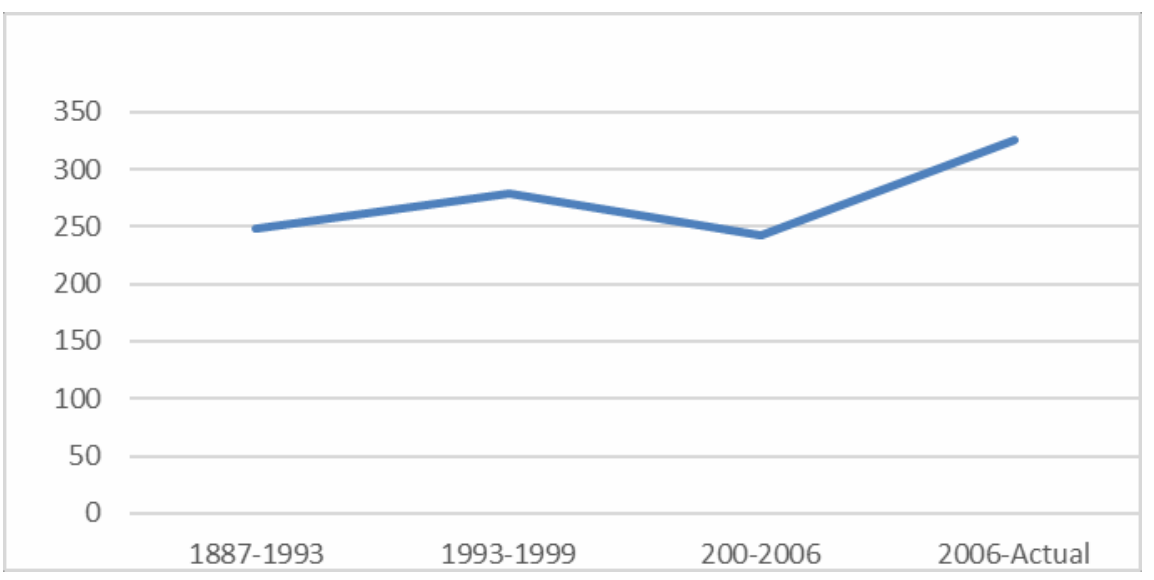

Al fragmentarse esta unidad educativa, todo el esfuerzo ganado y el tiempo invertido en lo que se había realizado para bien de la comunidad quedó atrás. Tampoco fue para los estudiantes la mejor alternativa asistir a la Universidad Nacional de Educadores, UNAE, la que abarcaba la formación docente en general, incluida la educación bilingüe en el país; menos aún si no se realizaba la carrera de EIB desde un real enfoque intercultural.

Este hecho se pudo corroborar mediante encuestas realizadas a los últimos alumnos formados en su institución, quienes en su mayoría preferían seguir estudiando en la misma comunidad de Quilloac. El resultado más notorio fue el escaso número de estudiantes que acudieron a la UNAE en un inicio. 


\section{Interculturalidad y su incorporación en la malla curricular de la Educación Bilingüe}

Ecuador como país pluricultural y multiétnico es un país rico en tradiciones donde las costumbres y prácticas sociales de las distintas regiones marcan vivencias distintas en cada pueblo, hecho que caracteriza a cada nacionalidad, como se denominan nuestras comunidades indígenas.

La nacionalidad Kichwa de Ecuador posee rasgos diversos, tanto los que provienen de una antigua matriz cultural, común a todos nuestros pueblos, como otros de características disímiles, desarrollados en las particularidades geográficas y sociales de las regiones de la Sierra y la Amazonía de nuestro país, en donde se asientan la mayor parte de las comunidades Kichwa hablantes. Ambas vertientes, la serrana y la amazónica, han luchado por mantener y recuperar sus rasgos identitarios, lo cual es muy marcado en la lengua particular de cada región e incluso en los matices de la lengua usada por las diferentes comunidades de las regiones norte, centro y sur del Ecuador, o los sociolectos que identifican una segunda lengua diferente en cada región de nuestro país. Esto conlleva a que el diseño curricular pensado para la formación docente en estas comunidades requiera de una especificidad propia para cada contexto en donde se aplique la educación intercultural bilingüe.

Esta lógica responde a que en la cosmovisión del mundo occidental juega más el concepto de lo lingüístico, mientras que en el mundo andino ocurre lo propio con el aspecto semántico, por lo que es importante considerar la concepción del tiempo y espacio del mundo andino, conceptos que responden a parámetros distintos del mundo occidental respecto de la educación, y aún más, en la enseñanza de la segunda lengua.

\section{Matriz Curricular del Instituto Pedagógico de Quilloac}

La Matriz Curricular para la formación de profesores de Educación Básica Intercultural Bilingüe del nivel tecnológico estaba basada en tres ejes de formación: Humana, Básica y Profesional. El estudio de la carrera se realizaba a través de cinco módulos de formación, en los que las materias de formación humana como lógica y ética, sicología y pedagogía, se encontraban orientadas a la EIB y al manejo y conservación del ecosistema. Los alumnos realizaban pasantías profesionales y el trabajo de graduación como requisitos para completar su carrera. 
En el nivel de educación básica, los alumnos realizaban el estudio de las lenguas de las nacionalidades, además de filosofía, cosmovisión e identidad cultural, como también castellano e inglés; e incursionaban en el campo de la inclusión y Educación Básica, donde realizaban sus proyectos a través de los módulos de investigación.

En el nivel de formación profesional, el estudiante iniciaba con las prácticas docentes y con el estudio de las Pedagogías Contemporáneas de Educación Intercultural Bilingüe, el Modelo de Sistema de Educación Intercultural Bilingüe (MOSEIB), además de realizar estudios de las didácticas de las lenguas y de otras materias aplicadas a la educación, como son matemáticas, ciencias, y otras optativas.

De acuerdo al análisis de la Matriz, la educación respondía a la realidad del contexto de la educación en donde se llevaba a cabo la EIB. Los estudiantes realizaban sus prácticas en las distintas comunidades de las regiones Sierra, Costa y Amazonía, donde los docentes controlaban su avance. Los estudiantes asistían al ISPEDIB-Q una vez cada mes para el control y estudio de sus investigaciones que llevaban a cabo para su tesis de graduación. Este currículo fue aprobado en el año 2005 por el CONESUP, para los alumnos de la carrera de Formación Básica Intercultural Bilingüe, Nivel Tecnológico, que desaparece con la formación de la nueva universidad de formación docente, UNAE, la que tomará a cargo la formación de estudiantes en EIB.

\section{Resultados de las encuestas}

Durante la fase exploratoria fue pertinente conversar abiertamente con los directivos de toda la Unidad Educativa Bilingüe de Quilloac. Se incursionó así sobre el quehacer de los alumnos docentes, mediante interacción dialógica con algunas alumnas del Instituto Pedagógico, quienes manifestaron su interés por seguir la formación docente y continuar con la licenciatura. De acuerdo al análisis de 22 de las 66 encuestas aplicadas se obtuvieron los resultados mencionados a continuación.

En cuanto a movilidad docente, la mayoría de los estudiantes del instituto realizaban las prácticas como alumnos docentes en escuelas localizadas en comunidades cercanas a la Provincia de Cañar y en otras provincias, por lo que manifestaron, de acuerdo a las encuestas que viajaban de 1 a 3 horas hacia el lugar de trabajo.

\section{4}

Pol. Con. (Edición núm. 26) Vol. 3, No 10, octubre 2018, pp. 3-18, ISSN: 2550 - 682X 
Respecto al conocimiento de la lengua Kichwa; de acuerdo al resultado se puede decir que en su mayoría los alumnos-docentes entrevistados conocían más el español, aunque el manejo de la lengua materna era bastante bueno, como se pudo corroborar con los resultados siguientes, en 22 encuestas de las 66 aplicadas; en un rango de 5 a 1, considerando el número mayor el número más más alto, como se aprecia en el cuadro.

\section{Gráfico 2. Uso de la Lengua kichwa y del español}

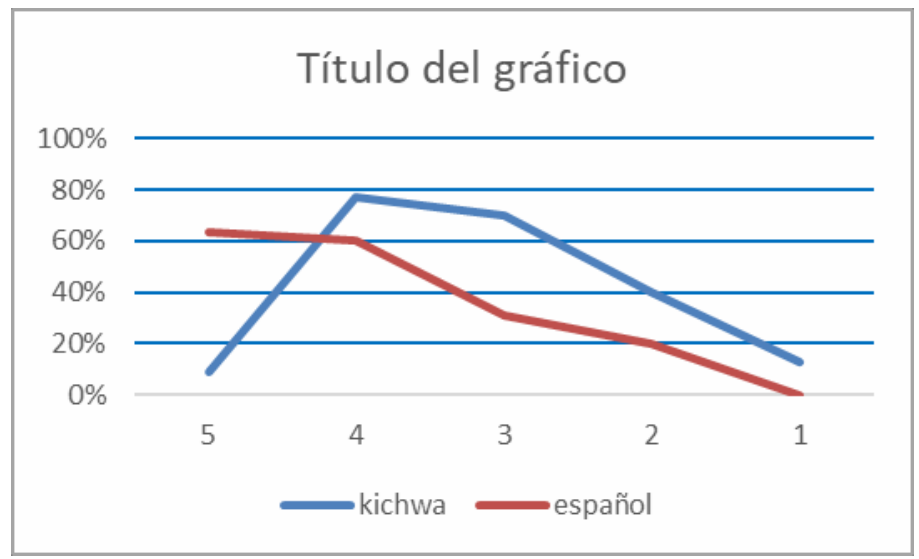

En cuanto al manejo de la lengua kichwa, un $9 \%$ se ubica en el rango más alto de 5; ellos utilizan la lengua; un $77.22 \%$ se ubica entre el $4 / 3$ y manifiestan que la utilizan bien y un $13 \%$ que no la manejan. En cuanto al uso del español por parte de los alumnos, en un rango de 5 a 1; considerando el numeral más alto el mejor, un $63.63 \%$ nos dicen que utilizan muy bien, mientras un $31,80 \%$ manifiestan que la usan bien y solo un 4,5\% manifiestan que no manejan la lengua segunda.

Esto nos hace ver que en algunos casos la segunda lengua, para ellos el español pasa a ser su primera lengua, y que la desapropiación de su lengua es también notoria. Los estudiantes manifiestan en un porcentaje aproximado a un $30 \%$ que presentan problemas en el aprendizaje del español. Las encuestan muestran que los alumnos presentan mayor dificultad en el aprendizaje de la lengua kichwa, casi en un 68\%, lo que corrobora de igual manera la desapropiación de su lengua. 
En cuanto al material de estudio proporcionado por el Ministerio de educación para la enseñanza bilingüe, solo un $13.60 \%$ manifiesta que es de mucha utilidad, mientras que un $86,36 \%$ dicen que es de poco uso o nulo. Esto nos demuestra que la conceptualización de la lengua materna no está bien manejada, mucho menos su cultura reflejada en la lengua segunda.

Las entrevistas abiertas aplicadas a tres maestras corroboran los datos de las encuestas, en cuanto a movilidad, situación escolar en el uso de la lengua y materiales. En el corpus recopilado a través de conversaciones y toma de notas en turnos, describieron tres situaciones distintas, con excepción de una en donde los alumnos conocían más la lengua kichwa. Esto demuestra que se debería considerar cada contexto de aprendizaje único, por lo que debería ser un factor tan importante para el análisis y aplicación de los materiales. Las entrevistas y transcripción de las notas y el proceso de escritura de la información se realizaron en la segunda lengua, el castellano y entre ellas manifiestan acerca del uso de la lengua Kichwa en la escuela:

“...que ellos no saben”; “...algunos nomás, otros no saben ni lo que hablan”; “...como yo doy a los del jardín, no saben nada"; '...todos entienden, pero no quieren hablar.” (Informante: alumna del último año del ISPEDIB-Q, 2014)

De acuerdo a las entrevistas semi- estructurada aplicadas con el fin de poder sistematizar el número de aspirantes que desean seguir su carrera; la mayoría manifiesta su afán por continuar una Licenciatura en EIB. Esto llevó a reflexionar que el Instituto Pedagógico de Quilloac, debería continuar con la formación docente, y que las políticas de educación bilinguie deberían ser flexibles para respetar estos centros educativos. La mayoría de sus directores manifestaron que seguirán luchando en este campo de la educación para tratar de conseguir por parte del gobierno central el apoyo para que el ISPEDIB-Q pudiera seguir con su labor de Formación Docente. Esto también debería ser un punto de análisis al respecto por las Universidades para apoyar a carreras de pos titulación para estos alumnos en la Licenciatura y formación de alumnos de cuarto nivel en EIB.

\section{Conclusión}

La formación educativa en EIB fue en muchas poblaciones del país el resultado de su plataforma de lucha por buscar una igualdad de oportunidades y su inclusión en el sistema educativo vigente. Dubet, citado en Tenti Fanfani (2008): menciona "La igualdad de 
oportunidades meritocráticas supone una igualdad de acceso", [...] no existe mayor justicia al reducir la diferencia de resultados en las categorías sociales al entrar los alumnos en la misma competición".

Por razones similares, frente a la problemática del Instituto Pedagógico de Quilloac se pensó en otro tipo de soluciones, como brindar a los maestros de EIB, formados en los diferentes institutos, la posibilidad de inclusión a nuestras universidades a través de un buen uso y manejo de su segunda lengua, para hacer de su educación un verdadero proceso inclusivo en el nivel superior. Esta propuesta podría ser viable sobre las bases del análisis de un trabajo de investigación y con el apoyo que la Universidad de Cuenca y de otras instituciones en el campo educativo podrían desarrollar mediante un nuevo proyecto de intervención, como apoyo a los profesores que se formaron en el Instituto Pedagógico de Quilloac.

Hubiera sido más asertivo de parte de nuestras universidades crear carreras de pos-titulación para facilitar a los alumnos que se formaron en los Institutos Pedagógicos Bilingües del país, una continuidad en formación bilingüe. Al analizar el último dato de los últimos alumnos egresados de Quilloac, con el número de alumnos que continuaron su carrera de EIB en la universidad de educadores, UNAE, no existe una relación paritaria. Para Delich (2014) "En la sociedad del conocimiento la educación es un bien público que compromete la existencia misma, la propia humanidad del Ser", hecho que tendría que traducirse en el respeto a las diferencias y a la manera como cada pueblo construye sus aprendizajes y sus propios conceptos de educación.

\section{Referencias Bibliográficas}

Delich, F. 2014. Sociedades Invisibles, La cultura de la ingobernabilidad en Latinoamérica. Gedisa: Buenos Aires

Fanfani T. (2002). En Peraza R. (comp.), 2008. Pensar en lo público: Notas sobre educación Estado. Ed. Aique Grupo Editor S. A.: Buenos Aires

Guamán F. M. (et .al.) (2011). La Nación Cañari y sus expresiones culturales/ Cañari Runakunapa kausaymapa Yachay. Museo Nacional del Indígena Ameriano. Ed. NMAI: Ecuador

Guber, R. (2011). Método, Campo y Reflexibidad. Siglo XXI: Buenos Aires 2008. (comp.) Pensar en lo público. Notas sobre educación y Estado. Aique Grupo Editor S. A.: Buenos Aires 
Fanfani, E. En Gluz N. y Jorge Arzate (coord.) s/f. Debates para la reconstrucción de lo público en educación: del universalismo liberal a "los particularismos" neoliberales. Universidad General Sarmiento

López, L. (2002). El multiculturalismo indolatinoamericano y la población indígena, Cochabamba, documento de trabajo. En Tedesco (2003). En Roxana Perazza (comp.) Pensar en lo público: Notas sobre la

Tiramonti G. (2008). Mutaciones en la articulación Estado- sociedad. En Peraza Roxana (comp.). Pensar en lo público: Notas sobre educación y Estado. Ed. Aique Grupo educación y el Estado. Buenos Aires: Ed. AIQUE

Verdugo A. et. al. (2010). La Nación Cañari y sus expresiones culturales/ Cañari Runakunapa kausaymapa Yachay. Museo Nacional del Indígena Ameriano. Ecuador: Ed. NMAI

Vélez C. (2008). Trayectoria de la Educación Intercultural en Ecuador. Revista Educación y Pedagogía, Vol. XX, No 52, Editor S.A.: Buenos Aires

Quinde, A. (2014) Informante: Director del ISPEDIB-Q. Quilloac, Cañar

Walsh C. (2012). Interculturalidad crítica y (de) coloniedad. Ensayos desde Abya Yala. Serie Pensamiento decolonial. Quito, Ecuador: Ediciones Abya Yala.

CRÉDITOS: Moreno, C. (2015). Fotografía: alumnas de Educación Básica, Unidad Educativa Quilloac. 